\title{
Live Demonstration: A Wearable EIT System for Hand Prosthesis Motion Controls
}

\author{
$\mathrm{Yu} \mathrm{Wu}^{1}$, Dai Jiang ${ }^{1}$, Richard Bayford ${ }^{2}$ and Andreas Demosthenous ${ }^{1}$ \\ ${ }^{1}$ Department of Electronic and Electrical Engineering, University College London, Torrington Place, London WC1E 7JE, UK \\ ${ }^{2}$ Department of Natural Sciences, Middlesex University, The Burroughs, London, NW4 4BT, UK \\ e-mail: yu.wu.09@ucl.ac.uk; a.demosthenous@ucl.ac.uk
}

\begin{abstract}
A wearable electrical impedance tomography system for hand prosthesis motion control is demonstrated. The system captures the user's hand motion by measuring the impedance alterations caused by muscle and bone movement inside the forearm. These impedance data are sent to an artificial neural network for motion classification which is then used to manipulate a hand prosthesis. During the live demonstration, a sensor band is put on a volunteers' forearm for data acquisition. After signal processing, hand gestures learnt by the neural network can be recognized and the same hand motion can be recreated through the hand prosthesis in real-time.
\end{abstract}

\section{INTRODUCTION}

Hand motion is one of the most important ways that humans interact with the outside world. Systems that are able to recognize these motions can create a seamless link between humans and machines to enhance the quality of life in countless applications. Many systems have been developed based on electromyography (EMG), or mechanomyography (MMG) principles [1]. This demonstration proposes a new hand motion capturing system that uses an alternative method called electrical impedance tomography (EIT).

EIT is a non-invasive technique that can be used to investigate the inner structural impedance distribution of an enclosed conductive object. An array of electrodes is placed around the subject under test (SUT) and by injecting a current, voltage potentials are developed corresponding to its inner impedance distribution that can be measured at SUT's surface through electrodes. Using tomographic reconstruction algorithms, measured results can be used to provide inner impedance distribution maps reflecting the cross-section area that the electrode array encloses. EIT has been widely used as an imaging technique in many medical research applications, such as brain activity detection and lung aeration monitoring. Hand motion is directly related to the physical movement of muscles and bones in the forearm causing characteristic impedance distributions. The proposed system shown in Fig.1 aims to detect these gestures by monitoring the temporal bioimpedance alteration occurring due to the movements. An artificial neural network (ANN) learns the bio-impedance pattern for each hand motion, and provides classification once the same pattern is subsequently detected. The classification can then be used to command a hand prosthesis and thus create a human-machine interface.

The system, as shown in Fig. 1, consists of a fully integrated EIT system using commercial ICs for high accuracy hand- gesture detection [2]. It captures the bio-impedance data and sends it to the ANN for feature learning and classification. Once a classification is given, the system sends pulse-widthmodulated pulses to a servo-motor based hand prosthesis in order to recreate the hand motion performed by the user.

\section{DEMONSTRATION SETUP AND VISITOR EXPERIENCE}

The proposed demonstration setup includes a wearable EIT system, shown in the bottom left of Fig. 1, a remote laptop computer running the ANNs and communicates with a hand prosthesis. A volunteer can experience this seamless link between humans and machines by wearing the EIT band around the forearm and performing a number of hand gestures. Once

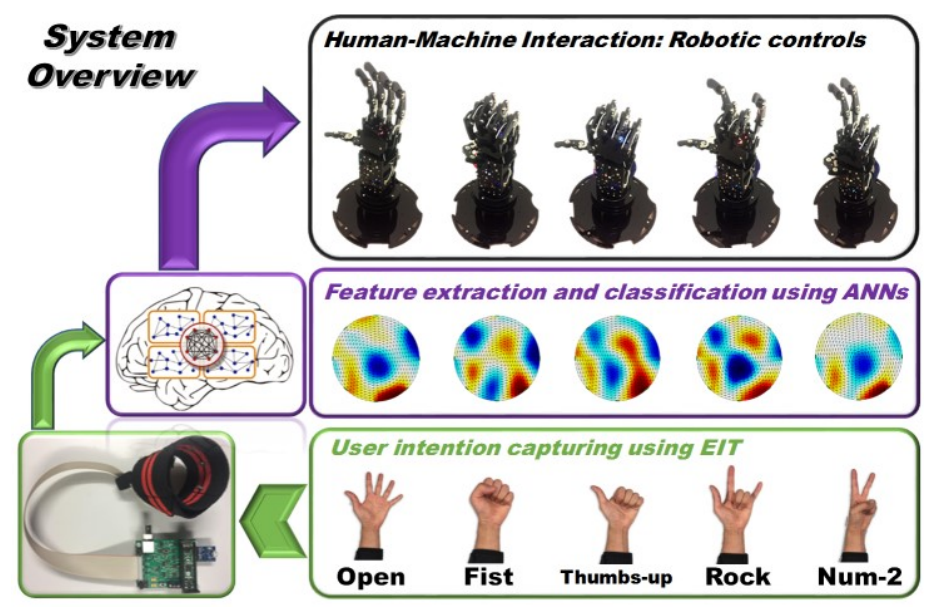

Fig. 1. Overview of the proposed human-machine interaction system for control of a hand prosthesis. Five hand motions are shown above as an example.

the ANN learns the bio-impedance patterns of the volunteer's hand gestures, they then can repeat these gestures and the same motion is recreated on the prosthesis hand in real time. At the same time, the inner bio-impedance map of the forearm is shown on the PC corresponding to the hand gesture performed for reference.

\section{REFERENCES}

[1] R. Woodward, S. Shefelbine, and R. Vaidyanathan, "Integrated grip switching and grasp control for prosthetic hands using fused inertial and mechanomyography measurement," Swarm/Human Blended Intelligence Workshop (SHBI), Cleveland, 2015, pp. 1-8.

[2] Y. Wu, D. Jiang, J. Duan, X. Liu, R. Bayford and A. Demosthenous, "Towards a high accuracy wearable hand gesture recognition system using EIT," ISCAS2018, Florence, Italy, 2018. 